\title{
La formación profesional básica, una alternativa para atender las necesidades educativas de los jóvenes en riesgo social
}

Ana Fernández-García

UNED (España)

José Luis García Llamas

UNED (España)

Marta García Pérez

Funcionaria de Educación Secundaria y Bachillerato 



\title{
La formación profesional básica, una alternativa para atender las necesidades educativas de los jóvenes en riesgo social
}

\section{Basic professional training, an alternative to address the edu- cational needs of young people in social risk}

\author{
Ana Fernández-García \\ UNED (España) \\ anafernandez@edu.uned.es \\ José Luis García Llamas \\ UNED (España) \\ jlgarcia@edu.uned.es \\ Marta García Pérez \\ Funcionaria de Educación Secundaria y Bachillerato \\ marta.garciaperez1@educa.madrid.org
}

Fecha de recepción: 9 de septiembre de 2018

Fecha de aceptación: 2 de noviembre de 2018

\begin{abstract}
Resumen
Este artículo se centra en analizar las necesidades de formación que poseen los jóvenes entre 15 y 26 años, que asisten al programa de Formación Profesional Básica, para continuar estudios y/o acceder a un empleo. El estudio realizado es exploratorio, descriptivo e inferencial desarrollado en la Comunidad de Madrid. Se ha elaborado un cuestionario ad hoc, validado por jueces expertos. Se analizaron los datos mediante métodos cuantitativos: descriptivos, inferenciales y multivariados. Los hallazgos han posibilitado el conocimiento de propuestas educativas para lograr una formación eficaz que permita a los jóvenes en riesgo social su integración en la sociedad mediante el apoyo de los implicados. Promover un enfoque formativo integral mediante una relación fuerte entre las instituciones formativas y lugares de trabajo, serían modos de avanzar en la igualdad de oportunidades en todos los estratos poblacionales de la sociedad y sobre todo en aquellos jóvenes más vulnerables.
\end{abstract}

Palabras clave: Juventud; Formación; Necesidades educativas; Investigación; Problemas Sociales. 
La formación profesional básica, una alternativa... - A. Fernández-García y otros

\begin{abstract}
This article is focused on analyzing training needs about youth among 15 and 26 years old, who attend the Basic Professional Training program, to continue studies and/ or to access to a job post. The made study is exploratory, descriptive and inferential type carried out in the Community of Madrid. A survey has been elaborated (ah doc), which was validate for expert judges. Quantitative methods were used to analysis data: descriptive, inferential and multivariate. The discoveries have let the direct knowledge of the real situations and contexts of the youth in social difficulty, like so the educational proposals which are carried out with the finality to get an efficient training which let youth in social risk to reach an integration in the society through the support of the involved. Promoting an integral and training approach close through a strong relationship among training institutions and labor places, they would be ways to go forward in the equal opportunities in all population strata and especially in youth more vulnerable.
\end{abstract}

Keywords: Youth; Training; Educational needs; Research; Social problems.

Para citar este artículo: Fernández-García, A.; García Llamas, J. L. y García Pérez, M. (2019). La formación profesional básica, una alternativa para atender las necesidades educativas de los jóvenes en riesgo social. Revista de humanidades, $\mathrm{n}$. 36, pp. 211-232. ISBN 1130-5029 (ISSN-e 2340-8995).

Sumario: 1. Introducción. 2. Metodología. 3. Resultados. 4. Conclusiones y discusión. 5. Referencias bibliográficas.

\title{
1. INTRODUCGIÓN
}

Este trabajo tiene por objetivo detectar las necesidades de formación de los jóvenes en dificultad social, especialmente de aquellos que son más vulnerables. La escasa formación adquirida se convierte en un hándicap relevante que condiciona su integración en la sociedad y el acceso al mercado laboral, en un escenario azotado por el desempleo "al coincidir la crisis económica (2008-2015) con su acceso a la edad laboral" (Vallejo, 2017, p. 1). Hoy en día, la transición del sistema educativo al mercado de trabajo aparece como algo casi imposible (Organización Internacional del Trabajo, 2016) con graves consecuencias para este grupo poblacional; por lo que es conveniente afrontar desde coordenadas diferentes las dificultades educativas y sociales existentes.

Con el objeto de fundamentar el estudio sobre la formación profesional de los jóvenes en dificultad social se ha realizado un análisis de artículos científicos publicados en revistas especializadas seleccionando investigaciones relativas al tema de estudio, siguiendo criterios de pertinencia y suficiencia y, teniendo en cuenta tres requisitos básicos: a) estar publicados entre los años 2013-2017; b) poseer una metodología cuantitativa, cualitativa o mixta y, c) ser de ámbito nacional y/o internacional. Todo el proceso metateórico elaborado se efectuó de forma inductiva y deductiva permitiendo definir las dos categorías más relevantes que caracterizan la 
formación profesional de los jóvenes en riesgo social, a saber: rasgos definitorios que caracterizan a los jóvenes en riesgo de exclusión social y análisis de los programas de formación que existen en nuestro país como una alternativa para aquellos alumnos que han abandonado el sistema educativo y desean reincorporarse al mismo. Se presentan a continuación las tendencias más significativas.

\subsection{Rasgos definitorios}

Respecto a los rasgos definitorios que caracterizan a estos jóvenes especialmente vulnerables, el análisis documental puso de relieve los siguientes aspectos: en general cuentan con problemas escolares, fracaso y abandono escolar temprano (Márquez y Gualda, 2014; Amores y Ricatto, 2015; Sarceda-Gorgoso et al., 2017); poseen insuficiente educación básica, una baja cualificación que les incapacita para acceder a un puesto de trabajo remunerado (Jurado et al., 2015; Vallejo, 2017); precariedad y falta de empleo (Montserrat y Melendro, 2017); presentan altos índices de desmotivación, estereotipos y falta de confianza respecto a la formación y al trabajo (Olmos y Mas, 2013); poseen carencias en habilidades sociales y laborales (Jurado, Olmos y Pérez, 2015); padecen problemas de acoso escolar, baja autoestima y aislamiento (Montserrat y Melendro, 2017; presentan dificultades económicas (Román, 2013); conviven en entornos familiares débiles y desfavorecidos, con presencia de desestructuración familiar y/o violencia en el hogar, con apoyos más frágiles (Márquez y Gualda, 2014; Vallejo, 2017); presencia de problemas de salud física y psicológica (abuso de substancias: drogas y alcohol, relaciones sexuales prematuras...) (Corbí y Pérez, 2013; Pérez et al., 2016); aumento del ocio que, en ocasiones, se caracteriza por un tiempo libre deconstructivo caracterizado por la inactividad, o por su contrario, mediante la participación en acciones no saludables y/o de alteración del orden público (vandalismo, hurtos...) (Fernández-García et al., 2015; Navarro-Pérez et al., 2015); existencia de desempleo generacional (Aparicio, 2014); índices de criminalidad (Sánchez-Castañeda, 2014); presentan escasas expectativas laborales y falta de oportunidades (Pérez, Velázquez et al., 2016); escaso conocimiento de los canales y herramientas facilitadoras de empleo (Jurado et al., 2015); y, presencia de violencia vecinal, viviendo en barrios marginados relacionándose con amistades peligrosas (García-Arjona, 2014).

Los jóvenes con mayor formación, ante la crisis económica descrita, que está sacudiendo nuestro país, entran en competencia con los jóvenes menos cualificados "al "arrebatar" puestos de menor rango a los jóvenes con menor formación" (Vallejo, 2017, p. 7); con el objetivo de acceder a un puesto de trabajo, dado que "ya no existen escenarios vitales sólidos, sino transiciones de movilidad, que permiten responder a las crecientes demandas de exibilidad" (Pérez et al., 2016, p. 53). Ello se traduce en una dificultad añadida a los jóvenes especialmente vulnerables donde $y$, ante el escenario descrito, la formación académica adquiere un papel muy relevante, pues constituye el soporte básico para que los jóvenes puedan adquirir conocimientos 
$\mathrm{y}$, mejorar sus cualificaciones para acceder a un puesto de trabajo en igualdad de oportunidades que el resto de los ciudadanos.

El fenómeno del abandono y fracaso escolar en España es controvertido y complejo (Amores y Ricatto, 2015); puesto que conlleva acercarse a la problemática realidad de la exclusión social y educativa. Destacamos que "el fracaso escolar, no es un término de nuevo cuño que defina una realidad emergente en nuestro sistema educativo actual, sin embargo, y lejos de quedar solventado se constata aún hoy, en nuestro país, su excesiva prevalencia con alrededor de un tercio del alumnado que finaliza y sale de la escuela sin los aprendizajes básicos" (Cutanda, 2014, p. 71). No obstante, "puede afirmarse que lo más preocupante es lo difícil que resulta alterar esas cifras en nuestro sistema" (Sarceda-Gorgoso et al., 2017, p. 80). Por ello se ha intentado mejorar esta situación, desde los años noventa del siglo pasado y se han desarrollado diferentes políticas educativas (con denominaciones que difieren según cada comunidad autónoma) con el objetivo común de proporcionar a los jóvenes excluidos del sistema escolar, una segunda oportunidad.

\subsection{Programas de formación}

En relación con los programas de formación debemos tener en cuenta las sucesivas reformas educativas generales existentes en España desde 1990: Ley Orgánica General de Ordenación del Sistema Educativo (LOGSE, 1990); la Ley Orgánica de Calidad de la Educación (LOCE, 2002) y la Ley Orgánica de Educación (LOE, 2006), que como indican Palomares y López (2013, p. 25), "han conllevado las dificultades propias de la inestabilidad legislativa y de los cambios constantes, dificultando en parte la consolidación de medidas consistentes y arraigadas garantes de una educación de calidad".

Como una nueva medida de atención a la diversidad y con el intento de evitar el fracaso y abandono escolar y favorecer el acceso a la vida laboral cualificada, en el año 2006, los Programas de Cualificación Profesional Inicial (PCPI) sustituyen a los Programas anteriores de Garantía Social (PGS). Los PCPI nacen en el contexto de la inclusión recogida en la Ley Orgánica 2/2006, de 3 de mayo, de Educación (LOE), destinados a una formación alternativa de alumnos mayores de 15 años que no habían obtenido el Graduado en Educación Secundaria Obligatoria (ESO). $\mathrm{Su}$ finalidad se centra en dar respuesta a un colectivo de jóvenes carentes de una formación inicial sólida. Persiguiendo que este colectivo alcanzase competencias profesionales que les facilitasen una inserción social y laboral satisfactoria, a la vez que ampliaran aquellas competencias que les permitiese proseguir estudios en los diferentes niveles de enseñanza.

No obstante, los Programas (PCPI) son sustituidos por la LOMCE (Ley Orgánica 8/2013, de 9 de diciembre, para la Mejora de la Calidad Educativa) estableciendo los Ciclos de Formación Profesional Básica (FPB) impartiéndose desde el curso 2014- 
2015. Las finalidades de los ciclos de formación básica se centran en preparar al alumnado para el ejercicio de actividades profesionales acordes con sus capacidades y expectativas personales. Asimismo, pretenden desarrollar su madurez personal mediante la adquisición de conocimientos y habilidades que les permitan participar en el trabajo, en actividades sociales y culturales integrándose y adaptando las actividades pedagógicas programadas a las características específicas del alumnado. "En la actualidad, estos programas de formación profesional básica se consideran un recurso para impulsar la empleabilidad de los jóvenes que se encuentran en riesgo de exclusión, puesto que favorecen su reincorporación al sistema educativo y aumentan sus posibilidades de inserción laboral" (Olmos y Mas, 2017, p. 261).

La Formación Profesional Básica abarca dos cursos académicos, ofreciendo a los jóvenes la formación necesaria para obtener una cualificación de nivel $1^{1}$ del Catálogo Nacional de las Cualificaciones Profesionales. Si el alumnado supera esta etapa educativa, se les facultará para acceder a Formación Profesional de Grado Medio. Además, podrán obtener el título de Graduado en Educación Secundaria Obligatoria (ESO) mediante la superación de una evaluación final en las condiciones que marca la Ley vigente. "Constituyen, pues, en cierto modo, una segunda oportunidad, dirigida a un alumnado que al haber finalizado su etapa obligatoria sin obtener el título de Graduado en Educación Secundaria Obligatoria, abandona el sistema educativo desprovisto de las competencias mínimas necesarias que le permitan transitar e insertarse en el mundo laboral, lo que les sitúa en una situación de enorme vulnerabilidad" (Cutanda, 2014, p. 70).

Está cualificación está regulada por el Real Decreto 127/2014, de 28 de febrero, donde quedan explícitos los aspectos específicos de la Formación Profesional Básica dentro de las enseñanzas de formación profesional del sistema educativo. Se fijan sus currículos básicos y se aprueban catorce títulos profesionales, a saber: Servicios Administrativos, Agrojardinería y Composiciones Florales, Artes Gráficas, Servicios Comerciales, Electricidad y Electrónica, Reforma y Mantenimiento de Edificios, Fabricación y Montaje, Cocina y Restauración, Alojamiento y Lavandería, Informática y Comunicaciones, Informática de Oficina, Peluquería y Estética, Carpintería y Mueble y Mantenimiento de Vehículos.

La Formación Profesional Básica posee una organización flexible, adaptándose a las singularidades del alumnado. Asimismo, en este nivel educativo se debe contar con la presencia de un equipo docente reducido que atienda a un mismo grupo de jóvenes, respetando los elementos educativos y el horario de los diferentes módulos impartidos. Respecto a la metodología, ésta posee un carácter integrador y globalizador, adaptándose a las necesidades de los alumnos. Es primordial que el alumnado pueda adquirir las competencias necesarias que les permitan el logro de

1 El nivel 1 del Catálogo Nacional de las Cualificaciones Profesionales integra los contenidos teórico-prácticos adecuados a los diversos campos profesionales y contribuye a que el alumnado adquiera las competencias del aprendizaje permanente. 
un aprendizaje permanente y continuo y les facilite la transición hacia la vida activa y ciudadana y su continuidad en el sistema educativo. "El diseño y el desarrollo de estos programas formativos - entendiéndose como una herramienta que ayuda a los alumnos a crecer desde el punto de vista competencial - facilitan la adquisición de dichas competencias (Olmos y Mas, 2017, p. 261).

\section{METODOLOGÍA}

El objetivo principal de esta investigación consiste en conocer las necesidades de formación de los jóvenes en dificultad social para acceder al empleo. De ahí que nos preguntemos: ¿en qué medida el programa de FPB (Formación Profesional Básica) proporciona al alumnado una adecuada formación para desempeñar un puesto de trabajo?; ¿Existen los medios y recursos necesarios (en el Estado Español) para proporcionar una adecuada formación para acceder al mundo del trabajo?; ¿Se encuentran, los implicados, satisfechos con su formación?; ¿En qué grado el alumnado está satisfecho con la ayuda y apoyo que reciben de los diversos profesionales e instituciones?; y, ¿Cuáles son sus perspectivas de futuro?

Los objetivos específicos que se han determinado hacen referencia a: analizar el perfil y características del alumnado que asiste al Programa de Formación Profesional Básica (FPB) y determinar el grado de satisfacción del alumnado que asiste a estos programas. modo:

Por su parte, las hipótesis de investigación quedan establecidas del siguiente

- La formación es un requisito indispensable para lograr un empleo, ya que a mayor formación existen mayores posibilidades de conseguirlo e insertarse en la sociedad.

- Los programas formativos FPB (Formación Profesional Básica) proporcionan una adecuada formación para el empleo de los jóvenes en dificultad social.

- La ayuda y apoyo que los jóvenes en riesgo social reciben de diversos profesionales e Instituciones es alta.

\subsection{Muestra}

La población objeto de estudio está formada por 14000 jóvenes entre 15 y 26 años en riesgo social que han cursado primero y segundo curso de Formación Profesional Básica (FPB) en el curso académico 2016/2017, tanto en centros educativos como en organizaciones dedicadas a la formación de los jóvenes en dificultad social en la Comunidad Autónoma de Madrid. El cálculo de la muestra nos dio un valor de 374 sujetos, a un nivel de confianza del $95 \%$, y un error de estimación de 0,05 . Sin embargo, la muestra final estuvo formada por 466 alumnos, cifra ligeramente más elevada que 
nos ha permitido afirmar que la muestra recogida justifica la población estudiada en la presente investigación. Las instituciones colaboradoras, han sido 13, pertenecientes a diferentes distritos y localidades de la Comunidad Autónoma de Madrid.

A continuación, se exponen las características principales de los individuos que componen la muestra de estudio: en relación con las características sociales, ambientales y educativas, los resultados obtenidos ponen de relieve que la muestra estuvo formada por 466 alumnos que han realizado el primer y segundo curso de Formación Profesional Básica (FPB), en la Comunidad Autónoma de Madrid. Las instituciones educativas participantes son: el 50\% Centros educativos (Institutos de Educación Secundaria, Centros de Formación Profesional...) y la otra mitad (50\%) está integrada por Asociaciones diversas que trabajan en formación con los jóvenes en dificultad social, de ocho comunidades autónomas españolas: Comunidad de Madrid (30,3\%); País Vasco (24,2\%); Castilla y León (18,2\%); Andalucía (15,2\%) y Galicia, Aragón y Asturias con un 3\% de representatividad, respectivamente.

En cuanto al nivel educativo, 44 sujetos han cursado el segundo curso del programa FPB, y 422 jóvenes cursan primero. De los cuales el $70 \%$ son hombres y el 30\% de mujeres. Sus edades están comprendidas entre los 15 y 26 años, siendo el porcentaje de alumnos más numeroso entre 15 y 18 años alcanzando un $80,3 \%$. A medida que la edad aumenta el porcentaje decae considerablemente, puesto que un $17 \%$ lo forman jóvenes cuyas edades están entre los 19 y 22 años; y solamente un $2,8 \%$ lo constituyen los estudiantes con edades entre los 23 y 26 años.

Las especialidades educativas elegidas por orden de preferencia son: Electricidad y electrónica (30,3\%); Peluquería y estética (13,3\%); Servicios administrativos $(13,3 \%)$; Mantenimiento de vehículos $(13,1 \%)$; Cocina y restauración $(11,7 \%)$; Informática y comunicaciones (7,3\%); Servicios comerciales (5,5\%); Fabricación y montaje (3,7\%); Carpintería y mueble (1,8\%).

En relación con el origen de procedencia, cabe reseñar la existencia de 26 nacionalidades diferentes. Más de la mitad son españoles, exactamente 314 sujetos representando el 67,4\%. Seguido de un 7,5\% de ecuatorianos, República Dominicana $(5,4 \%)$; Colombia (3,9\%); Marruecos (3,6\%); Rumanía (3,2\%); Perú (3\%); Bolivia $(1,1 \%)$; Paraguay $(0,6 \%)$; Argentina $(0,4 \%)$; Brasil $(0,4 \%)$; Chile $(0,4 \%)$; Alemania $(0,2 \%)$; Bangladesh $(0,2 \%)$; Bulgaria $(0,2 \%)$; Camerún $(0,2 \%)$; China $(0,2 \%)$; Cuba $(0,2 \%)$; El Salvador $(0,2 \%)$; Filipinas $(0,2 \%)$; Finlandia $(0,2 \%)$; Guinea Ecuatorial $(0,2 \%)$; Honduras $(0,2 \%)$; México $(0,2 \%)$; Polonia $(0,2 \%)$; y Ucrania $(0,2 \%)$.

\subsection{Instrumentos}

El instrumento utilizado para la recogida de información ha sido el cuestionario elaborado "ad hoc" y denominado "formación para el empleo," aplicado a jóvenes entre 15 y 26 años. 
El objetivo principal del instrumento ha consistido en determinar el perfil y características del alumnado que asiste al Programa de Formación Profesional Básica (FPB). El cuestionario elaborado está formado por cuatro dimensiones, en consonancia con el planteamiento inicial, definiéndose un objetivo específico para cada una de ellas, a saber: Formación (Analizar los motivos y razones por las que está matriculado); Empleo (Descubrir si estos sujetos trabajan o han trabajado); Expectativas de futuro (Explorar de quién o quiénes han recibido apoyo); y Buenas prácticas, organizaciones y entidades(Identificar cuáles son sus expectativas de futuro).

Respecto a la construcción del cuestionario, para la dimensión Formación se han elaborado cuatro preguntas, dos de ellas de escala dicotómica (si/no) y las restantes requieren el grado de acuerdo, a seleccionar en una escala de 1 a 10 (1 valoración mínima, 10 valoración máxima). La dimensión Empleo está formada por dos preguntas: la primera de ellas de respuesta dicotómica (si/no) y la segunda requiere el grado de acuerdo en una escala de 1 a 10. La dimensión Expectativas de futuro está formada por una pregunta que contiene ocho ítems, a responder en una escala de 1 a 10. La última dimensión hace referencia a las Buenas prácticas, organizaciones y entidades y está formada por dos preguntas. La primera de ellas está formada por siete ítems con tres opciones de respuesta que determinan el grado de ayuda que reciben los jóvenes (me ayudan actualmente; me han ayudado en el pasado; nunca me han ayudado). La última pregunta está formada por trece ítems cuya respuesta consta de cuatro opciones (nada, poco, bastante, mucho).

Esta herramienta reúnes las características y requisitos técnicos de validación y fiabilidad. La validación se realizó a través de la validez de contenido con preguntas directas sobre la valoración de los ítems y su ubicación en los campos respectivos. Fue contestada por 20 expertos en los campos de la docencia y la metodología de investigación. Los resultados han sido muy positivos, con unos valores superiores en la valoración de los ítems frente a su ubicación. Por su parte, la fiabilidad del cuestionario completo se llevó a cabo mediante el coeficiente Alfa de Cronbach, que se considera el más adecuado para este tipo de instrumento de recogida de datos. El valor de 0,891 , refleja un alto grado de concordancia interna entre los ítems que lo integran.

La aplicación del cuestionario se realizó de forma directa en los Centros Educativos y Asociaciones donde se desarrollan los Programas formativos de FPB (Formación Profesional Básica) durante los meses de enero, febrero y marzo de 2017. Se han recogido un total de 466 cuestionarios de los cuales 233 pertenecen a los alumnos de centros escolares y los restantes 233 fueron recogidos en las asociaciones que han participado en la investigación. Toda la recogida de información ha sido muy costosa debido a las complejas características que reúnen los participantes.

\subsection{Procedimiento de análisis}

Como vía de trabajo se ha utilizado la metodología cuantitativa. El proceso de análisis de los datos cuantitativos se llevó a cabo con el programa SPSS ((Statistical 
Package for the Social Sciences) 22.0 empleando diferentes estadísticos en cada etapa:

Análisis descriptivo univariado. Permite establecer, de forma básica, los perfiles de los participantes en cuanto a sus rasgos personales (sexo, edad, nacionalidad, institución, curso académico...); análisis inferencial bivariado que cumple una función complementaria a los descriptivos, ya que mejoran el análisis de los datos ofreciendo una visión más global de los mismos. Para ello se ha fijado un margen de error en el 0,05 ; lo que equivale a un nivel de confianza del $95 \%$. Se han realizado los siguientes procesos: Contrastes mediante el ANOVA; contrastes mediante la prueba "t." de Student o la "z" de Fisher; y contrastes mediante la prueba de Ji Cuadrado.

Análisis multivariante. Por medio de este análisis podemos depurar aún más el cuestionario de recogida de datos. Para ello, se han analizado los datos que ponen de relieve diferencias significativas entre los grupos del estudio. Los procedimientos utilizados para el análisis son los siguientes: El análisis de clúster o conglomerados y el análisis de segmentación.

El análisis de clúster o conglomerados, conocido también como Reconocimiento de Patrones es una técnica de análisis exploratorio de datos para resolver problemas de clasificación. Es una técnica exploratoria, no inferencial, descriptiva y sin variables dependientes. Su objeto consiste en ordenar objetos (variables con nivel de medida ordinal en nuestro estudio) en grupos de forma que el grado de asociación/similitud entre miembros del mismo clúster sea más fuerte que el grado de asociación/similitud entre miembros de diferentes clústeres. Su finalidad es la formación de grupos. Para realizar este análisis se ha utilizado el programa estadístico SPSS 22.0. Los resultados se presentan mediante "dendogramas", que son representaciones gráficas de las clasificaciones jerárquicas.

Mediante el análisis de segmentación se creará un modelo de clasificación basado en árboles ("árbol de decisiones"). Este procedimiento pronostica valores de una variable (criterio) dependiente basada en valores de variables independientes (predictores). De esta manera proporciona herramientas de validación para análisis de clasificación exploratorios y confirmatorios.

Se ha utilizado el método de crecimiento CHAID (CHi-square Automatic Interaction Detection). En cada paso CHAID presenta la interacción más fuerte de la variable independiente con la variable dependiente fundiéndose las que no sean significativas. Este método examina todas las divisiones posibles y muestra la salida mediante un "árbol" clasificatorio de fácil interpretación y comprensión. Para realizar este análisis se ha utilizado el programa estadístico SPSS 22.0. El programa SPSS detectará automáticamente las interacciones mediante Ji cuadrado. Así mismo, los valores de significación se corrigen mediante el método de Bonferroni. 


\section{RESULTADOS}

Para la dimensión de formación para el empleo, los resultados ponen de relieve que el $48,1 \%$ del alumnado estudian en el centro educativo con interés para obtener el graduado en ESO (Educación Secundaria Obligatoria), frente al 30,5\% que desea obtener un certificado de profesionalidad para acceder al mercado laboral y un 21,5\% que prefiere seguir estudiando FP (Formación Profesional) para obtener un mayor nivel académico.

\begin{tabular}{|l|c|c|c|}
\hline \multicolumn{4}{|c|}{ ESTUDIO EN ESTE CENTRO PARA... } \\
\hline & Frecuencia & $\begin{array}{c}\text { Porcentaje } \\
\text { válido }\end{array}$ & $\begin{array}{c}\text { Porcentaje } \\
\text { acumulado }\end{array}$ \\
\hline Obtener el graduado ESO & 224 & 48,1 & 48,1 \\
\hline Seguir estudiando Formación Profesional (FP) & 100 & 21,5 & 69,5 \\
\hline Obtener un certificado de profesionalidad & 142 & 30,5 & 100,0 \\
\hline Total & 466 & 100,0 & \\
\hline
\end{tabular}

Tabla 1. Estudio en este centro para...

Se realizó también un análisis de contingencia relacionando las variables Institución y Estudio en este centro para ... se pudo constatar que existen diferencias en cuanto al propósito de cursar esta formación. El grado de significación alcanzado mediante el estadístico chi-cuadrado obtuvo un valor de $(, 013)$. El alumnado que asiste a los centros educativos otorga mayor importancia a continuar sus estudios en Formación Profesional (un 25\% desea seguir estudiando FP frente al 18\% del alumnado que estudia en Asociaciones). Así mismo, el que asiste a las asociaciones desea obtener en un $37 \%$ el certificado de profesionalidad que le permita trabajar, frente al $25 \%$ que lo desea en los centros educativos. Por otra parte, en ambas instituciones el alumnado quiere obtener el graduado en ESO en un alto porcentaje ( $51 \%$ y $46 \%$, respectivamente). De todos modos, se pone de relieve su baja formación.

Respecto a la pregunta: Durante la semana, ¿Cuántas horas dedicas al estudio?; el $28,5 \%$ del alumnado declara no estudiar nada. Entre 1 y 3 horas, se ubican el $47 \%$. El 13,9\% afirma que estudia entre 4 y 6 horas; el 4,9\% entre 7 y 9 horas y finalmente un 5,6\% declara que estudia más de 10 horas durante la semana. Cabe considerar que el alumnado al responder a la pregunta ha manifestado más horas de las que en realidad le ha dedicado al estudio dado que incluyen el tiempo que están en las aulas. No obstante, casi un 30\% de los encuestados ha manifestado no estudiar absolutamente nada, fuera del horario educativo.

Al preguntarle si: ¿Te gustan los estudios que realizas?; el 90,8\% afirman que sí le gustan los estudios que está realizando, frente al 9,2\% que lo niega. Se han cruzado, mediante un análisis de contingencia, las variables: Institución y Curso 
académico, con la variable ¿Te gustan los estudios que realizas? Se obtuvo un grado de significación mediante chi-cuadrado de valor $(, 000)$ para ambos cruces, lo que pone de relieve que el alumnado que estudia en las asociaciones se encuentra más satisfecho con los estudios que están realizando, que aquellos otros jóvenes que estudian en centros educativos. Aspecto que puede deberse al hecho de que el alumno que cursa sus estudios en asociaciones lo ha escogido por propia elección, frente al alumnado de los centros educativos que su asistencia puede verse condicionada por sus padres.

El alumnado de primer año manifiesta que sí les gustan los estudios en un $92,9 \%$ y no a un 7,1\%. Mientras que a los de segundo curso les gustan los estudios, al $70,5 \%$ y no, a un $29,5 \%$. Aspecto que puede deberse a la ilusión que les trasmite el primer año y al cansancio del segundo curso en jóvenes menos atraídos por la formación.

Cuando se les pregunta, ¿por qué te has matriculado en estos estudios?; el ítem para aprender un oficio obtiene la media más alta con un 8,54 (en una escala de 1 a 10, donde 10 es la valoración más alta) le sigue con una media de 7,64, el ítem, quiero saber más. La media de 5,38 la obtiene el ítem, relacionarme con los compañeros $\mathrm{y}$, una media de 4,80 por relacionarme con los profesores. Variables que han obtenido medias bajas son: no tengo otra cosa mejor que hacer, con 2,96 y, me han obligado, con 2,45, lo cual refleja que están en desacuerdo con estas atribuciones.

\begin{tabular}{|l|c|c|}
\hline \multicolumn{3}{|c|}{ ¿POR QUÉ TE HAS MATRICULADO EN ESTOS ESTUDIOS? } \\
\hline & Media & $\begin{array}{c}\text { Desviación } \\
\text { estándar }\end{array}$ \\
\hline Para aprender un oficio & 8,54 & 2,267 \\
\hline Quiero saber más & 7,64 & 2,544 \\
\hline Por relacionarme con los/as compañeros/as & 5,38 & 2,917 \\
\hline Por relacionarme con los/as profesores/as & 4,80 & 2,973 \\
\hline No tengo otra cosa mejor que hacer & 2,96 & 2,935 \\
\hline Me han obligado & 2,45 & 2,506 \\
\hline
\end{tabular}

Tabla 2. ¿Por qué te has matriculado en estos estudios?

Mediante el análisis clúster se obtienen grupos de ítems por afinidad en las variables vinculadas a las relaciones sociales: por relacionarme con los compañeros y por relacionarme con los profesores. En las variables: me han obligado y, no tengo otra cosa mejor que hacer. Y en las variables: quiero saber más y, para aprender un oficio. Todas ellas hacen referencia al aprendizaje y formación que recibe este alumnado en los centros.

En cuanto a los estudios que están realizando los resultados ponen de relieve que están satisfechos, alcanzando una media de 8,18 (en un recorrido de 1 a 10), y 
que su familia le anima a que los finalices logrando una media de 8,66; y si cree que al finalizar los estudios estará más preparado para trabajar que alcanza una media de 8,64; por último, la relación con sus profesores obtiene una media de 7,50. Para cada una de estas cuatro variables dependientes se ha realizado un árbol de decisiones (segmentación) que refleja las variables independientes más relevantes que definen cada categoría. Para la pregunta, Tu familia te anima a que finalices los estudios, la variable que ha mostrado mayor afinidad ha sido, mi madre, reflejando un mayor apoyo de la figura maternal. Así mismo, le otorgan valor a la relación que mantienen con los profesores explicada por el apoyo que les ofrecen para finalizar con éxito su formación. También es importante la familia por el apoyo que reciben de los padres para finalizar con éxito los estudios.

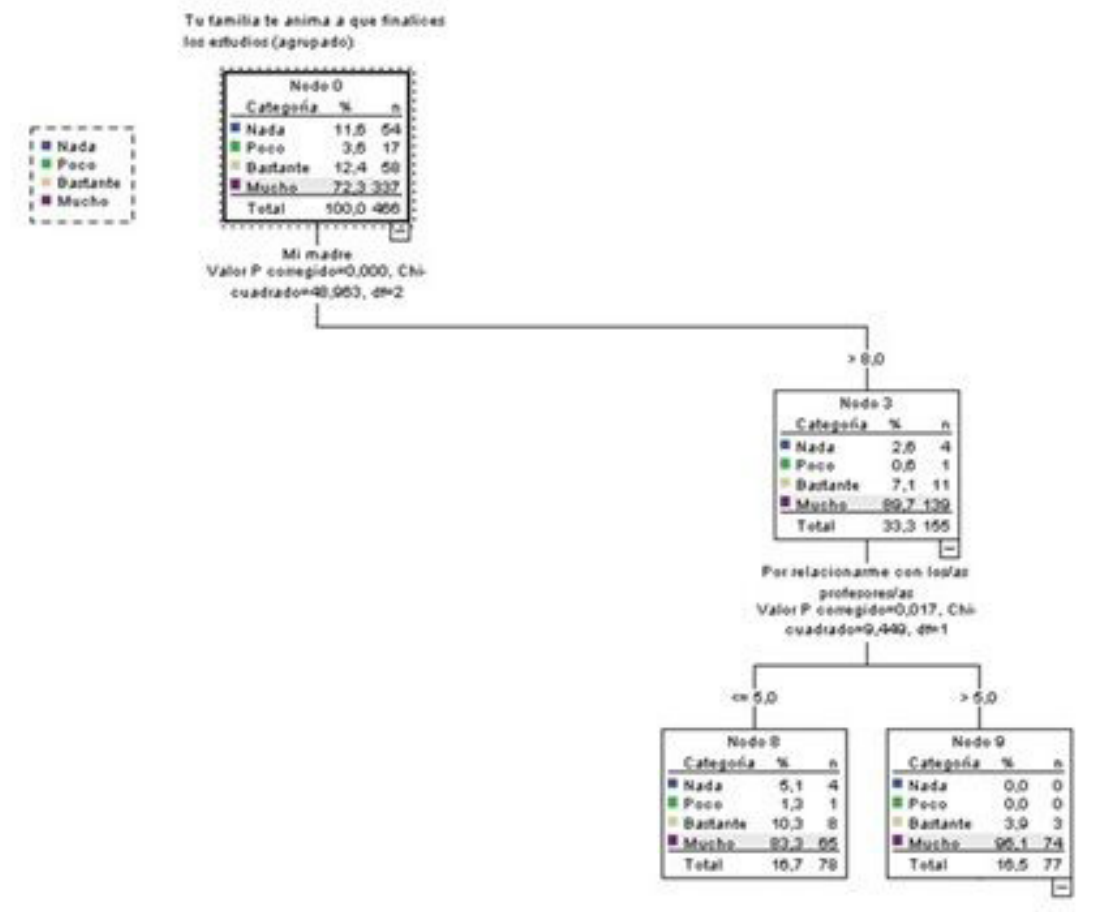

Gráfico 1. Árbol de decisiones. Tu familia te anima a que finalices los estudios

Respecto a la variable cuando finalice los estudios estaré más preparado para trabajar, la variable predictora que mejor define esta categoría es, para aprender un oficio. Insisten en la necesidad de buscar un trabajo en el extranjero, aspecto que denota la percepción de estos jóvenes ante la falta de oportunidades que existen en España para conseguir un trabajo. Así mismo, valoran que los educadores/ profesionales los apoyen para conseguir un trabajo. 
Por su parte, Quiero saber más... es la variable más afín a, ¿Estás satisfecho con los estudios que estás realizando? Así mismo, los jóvenes manifiestan en un $75,1 \%$ que les gustan mucho las actividades que realizan en los centros educativos y que los educadores/profesionales les animen a conseguir un trabajo. El alumnado otorga un valor medio a la variable aprendo cosas útiles y prácticas, al tiempo que sus familiares los animan a asistir a los centros educativos. Como valor negativo, los encuestados sienten que sus compañeros no les prestan apoyo en el estudio.

Para la variable, Cómo calificarías tu relación con los profesores, la variable independiente que mejor la predice es la propia relación social y profesional que establecen con los docentes, y que el $61,9 \%$ del alumnado lo ha valorado en bastante y mucho. Los jóvenes encuestados consideran que los educadores/profesionales les apoyan para conseguir un trabajo, les ayudan en el estudio y a participar en actividades lúdicas y prácticas.

En cuanto a las expectativas de futuro que tienen frente a su vida calificadas de 1 a 10 ( 1 es la calificación mínima y 10 es la máxima) han señalado varios aspectos positivamente, tales como: algún día me gustaría formar mi propia familia, con una media de 8,39; seguido de la variable, me gustaría independizarme económicamente, con una media de $8,18 \mathrm{y}$, pienso continuar estudios obtiene la media de 7,50. Así mismo, no descartan la posibilidad de buscar trabajo en el extranjero, puesto que ha alcanzado una media de 6,19 y con menor puntuación estaría la variable, colaborar con alguna ONG (Organización No Gubernamental) con una media de 4,92.

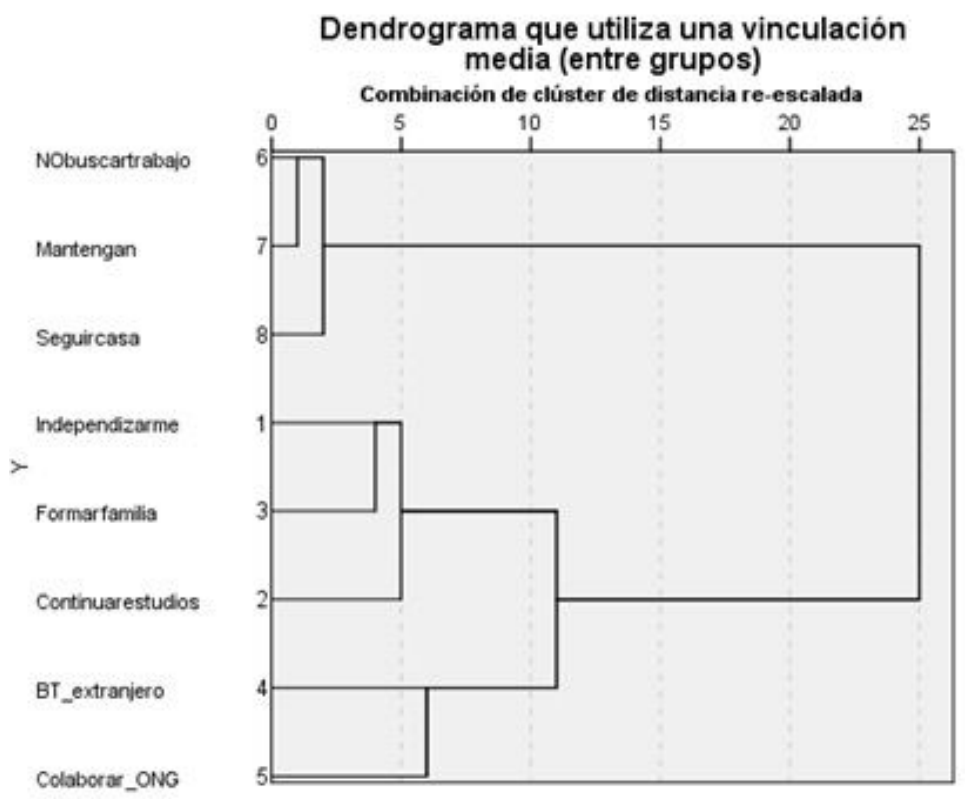

Gráfico 2. Dendograma. Expectativas de futuro 
La formación profesional básica, una alternativa... - A. Fernández-García y otros

Respecto a los centros educativos, los jóvenes valoran (bastante-mucho 64,2\%), que en ellos aprenden cosas útiles y prácticas. Con un porcentaje similar (64,2\%) que los educadores y profesionales les ayudan en el estudio y les animan a participar (56\%). Sin embargo, otros jóvenes son más pesimistas en relación con la ayuda que les prestan los profesionales en aspectos como: conseguir un trabajo $(57,7 \%$ nada y poco) y para realizar prácticas en centros externos ( $58,8 \%$ nada y poco).

\section{GONGLUSIONES Y DISGUSIÓN}

Teniendo en cuenta los planteamientos del estudio se concluye que el perfil del alumnado participante en su gran mayoría son hombres. Sus edades están comprendidas entre los 15 y 26 años, aunque el porcentaje más numeroso se sitúa en la franja entre 15 y 18 años. Proceden de 26 nacionalidades diferentes, si bien predominan los jóvenes españoles.

En cuanto a los motivos y razones por los cuales están matriculados y realizan estos estudios, casi la mitad del alumnado el $(48,1 \%)$ tiene interés en obtener el graduado en la ESO, frente a un $30,5 \%$ que aspira a conseguir el certificado de profesionalidad para acceder a un puesto de trabajo, y un $20 \%$ desea continuar estudios en Formación Profesional.

Estos resultados, son muy similares a los encontrados en otros estudios como el llevado a cabo por García (2014) donde han analizado las razones por las que los jóvenes deciden cursar estos programas y coinciden con nuestro estudio dado que los jóvenes están interesados en obtener el Graduado Escolar. No obstante, "la nueva FPB [...] elimina la posibilidad de obtener de forma directa el graduado de la ESO" (Tarabini y Montes, 2015, p. 5); aspecto que deben lograr mediante la superación de la reválida establecida. Asimismo, sí da acceso a la formación profesional de grado medio sin la necesidad de poseer el graduado escolar.

Le otorgan también un alto valor a esta formación en la medida que les posibilita el acceso a un empleo (Olmos y Mas, 2013). No obstante, los autores Adame y Salvà (2010), relatan que algunos jóvenes cursan estos estudios sin tener muy clara su utilidad y expresan que los cursan porque no tienen otra cosa mejor que hacer.

Al identificar cuáles son sus expectativas de futuro, los jóvenes han manifestado en primer orden que algún día les gustaría formar su propia familia y ser independientes y autónomos económicamente. Con relación a detectar el grado de satisfacción con su formación actual y con la ayuda que reciben de diversas Instituciones, se puede considerar que el alumnado encuestado está satisfecho y aprecia la ayuda que le ofrecen las instituciones a las que asiste $y$, valoran muy positivamente tanto a los centros educativos como a las asociaciones que imparten el programa de FPB. Aspecto corroborado en otras investigaciones (González y Porto, 2013) al afirmar 
que el alumnado está satisfecho con estos programas educativos percibiendo que lo que están aprendiendo tiene validez futura.

En general destacan la necesidad de una sólida y adaptada formación teórica y práctica llevada a cabo con una óptima intervención socioeducativa por todos los docentes y agentes implicados, con el fin de lograr su integración en el contexto en el que vive (social, cultural, humano, económico y laboral).

Cabe resaltar que una formación inicial sólida (teórica y práctica) constituye la base más importante, para que los jóvenes construyan aprendizajes que garanticen una continuidad en su proceso de formación y su desarrollo profesional y vital. Reiteran que la educación y la formación permiten mejorar la calidad de vida de las personas, debido a su importante papel sobre las oportunidades laborales. Se destaca que cuanto menor es el nivel de estudios de una persona, mayor es el riesgo de que pueda padecer problemas graves de salud, de inestabilidad laboral, de alojamiento precario, lo que les puede llevar a mayor probabilidad de sufrir exclusión.

En relación con la primera hipótesis planteada, que alude a, si la formación es un requisito indispensable para lograr un empleo, ya que a mayor formación existen mayores posibilidades de conseguirlo e insertarse en la sociedad; cabe reseñar que en esta investigación se ratifica esta afirmación, puesto que los individuos que poseen un mayor nivel educativo forman parte, durante más tiempo, de la población activa y reducen su presencia en la población desempleada.

Aspecto que se ratifica en diversas investigaciones, tal como en Melendro (2014, pp. 41) al concluir que "el efecto positivo de la educación sobre la empleabilidad juvenil también ha aumentado durante la crisis actual", puesto que finalizar los estudios reduce el riesgo de sufrir desempleo. La educación juega un papel importante en la lucha contra la exclusión social, ayudando a que los jóvenes logren cualificaciones profesionales y mejoren sus habilidades básicas. Por ello, proporcionar a la juventud una formación básica y profesional para un empleo es la mejor garantía de darles una oportunidad para la empleabilidad.

Respecto a la segunda hipótesis planteada, relativa a si los programas formativos FPB (Formación Profesional Básica) proporcionan una adecuada formación para el empleo de los jóvenes en dificultad social, cabe destacar que en el estudio realizado el alumnado está satisfecho con esta formación, aspecto que resulta altamente significativo si tenemos en cuenta que el compromiso y la motivación de los alumnos son factores esenciales para culminar con éxito los estudios. Este programa (FPB), denominados de segunda oportunidad, "para un colectivo de jóvenes expulsados y rechazados del propio sistema educativo" (Olmos y Mas, 2017, p. 263), no puede en ningún caso reproducir el modelo escolar en el que han fracasado con anterioridad los jóvenes. Ante ello consideramos que la flexibilización del currículum es un elemento clave, dado que ofrece nuevas posibilidades para el alumnado más desaventajado. 
Así mismo, es necesario que se inserten en la vida del centro y en el contexto en el que se encuentran.

La optimización de recursos públicos y privados existentes para su desarrollo debe ser un eje central, desde el fortalecimiento de las relaciones y sinergias entre diferentes instituciones; puesto que el trabajo entre todos los implicados precisa y requiere ser interdisciplinar e interinstitucional (Melendro, 2014; Pérez, Poza-Vilches y Fernández-García, 2016), para que se pueda alcanzar el éxito en la intervención. Actuaciones aisladas conducen a una segmentación de éstas sin un nexo de trabajo común y a una reiteración de recursos. Sobre el uso de espacios y recursos se confirma que sean abiertos y flexibles para el desarrollo de las actividades.

Igualmente, los mencionados programas formativos sólo pueden tener éxito si éstos son flexibles, transferibles, eficientes y si los jóvenes y profesionales implicados están involucrados, asumiendo un papel activo y responsable.

Por último, la tercera hipótesis planteada hace referencia a si la ayuda y apoyo que los jóvenes en riesgo social reciben de diversos profesionales e Instituciones es alta. Cabe reseñar que, en los resultados obtenidos, mediante las diferentes técnicas de investigación empleadas, se reitera la necesidad de una mayor dotación de recursos materiales y humanos para atender a esta población, prestando atención a su gestión positiva.

Por otra parte, se confirma que el profesorado es un factor esencial para el éxito del alumnado, de ahí lo importante que es la formación continua y actualizada de este colectivo para que mejore su intervención educativa. También se deben tener en cuenta otros factores como el grado de implicación, compromiso, coordinación y participación de todos los agentes implicados, para que toda intervención socioeducativa pueda lograr el éxito esperado en la formación de los jóvenes vulnerables evitando duplicidades. Por lo general, los alumnos desean que estos profesionales diseñen metodologías directas e individualizadas y realicen un seguimiento personalizado para atender las necesidades y demandas educativas y socio laborales singularizadas. Nuestra investigación ha dado como resultado que los jóvenes respetan y valoran a los profesores, poniendo de relieve su importancia en el proceso formativo. Aspecto que coincide con la investigación de Montserrat y Melendro (2017), cuando ponen de relieve "la buena valoración de los adolescentes, especialmente de los más dañados, con problemáticas más complejas y graves, hacia sus educadores, su profesionalidad y las habilidades y competencias que describen como más signicativas" (p. 129).

Con relación al aspecto laboral, la crisis económica mundial ha generado menos oportunidades de trabajo, especialmente en esta tipología de jóvenes y, sobre todo, en los que carecen de experiencia. Así mismo, en aquellos países con sistemas educativos de poca calidad y que poseen débiles vínculos con el mercado de trabajo, carecen de un adecuado proceso de transición de la escuela al trabajo, tal y como lo expresa 
Vallejo (2017) al afirmar "la incapacidad mostrada por nuestras instituciones para afrontar los desajustes formación/empleo sufridos por la juventud española" (p. 12).

Los jóvenes son sujetos de derechos, y no pueden ser considerados como objetos problemáticos cuya dificultad reside en su incapacidad individual para adaptarse a las condiciones que establece el mercado de trabajo. Al contrario, hay que ayudarles a establecer su propio itinerario formativo y laboral, mediante una atención individualizada e interviniendo de manera global, trabajando en su empleabilidad, tanto para acceder a un trabajo como para mantenerse en el mismo.

Por todo ello, promover un enfoque de formación integral junto con las políticas públicas y laborales mediante una relación fuerte entre las instituciones formativas y lugares de trabajo laboral serían modos de avanzar en la igualdad de oportunidades en todos los estratos poblacionales de la sociedad y sobre todo en aquellos jóvenes más vulnerables.

Ante ello, los gobiernos y las diferentes administraciones deberían apoyar a los jóvenes con medidas más sólidas en materia de educación para la inserción sociolaboral, con la finalidad de otorgarles nuevas oportunidades y, con ello, lograr una comunidad cohesionada fortaleciendo el reemplazo generacional.

En cuanto a las limitaciones de la presente investigación, destacamos que en las instituciones educativas sólo se ha recogido la información de los estudiantes y, teniendo en cuenta sus características y difícil acceso, el cuestionario formación para el empleo ha sido muy reducido, obviando preguntas de carácter cualitativo y abierto que pudiesen enriquecer la información obtenida.

Asimismo, se torna necesario seguir investigando en aras de descubrir si los Programas educativos de Formación Profesional Básica proporcionan las competencias y capacitación necesaria para una óptima incorporación laboral de un sector poblacional especialmente vulnerable. Para ello, aplicar el cuestionario a jóvenes de todo el territorio español, permitiría elaborar un estudio a nivel nacional y comprobar si existen diferencias significativas entre Comunidades Autónomas en la implementación de esta formación. Ello enriquecería notablemente los resultados obtenidos y, en general, un mayor conocimiento de la FPB.

\section{REFERENCIAS BIBLIOGRÁFICAS}

Adame Obrador, María Teresa y Salvá Mut, Francesca (2010). Abandono escolar prematuro y transición a la vida activa en una economía turística: el caso de Baleares. Revista de Educación, n. 351, pp. 185-210.

Amores Fernández, Javier y Ricatto Real, Maximiliano (2015). De los Programas de Cualificación Profesional Inicial (PCPI) como medida de prevención del fracaso escolar a la Formación Profesional Básica. Un estudio sobre el éxito y fracaso de 
La formación profesional básica, una alternativa... - A. Fernández-García y otros

alumnos en riesgo de exclusión educativa en Educación Secundaria. Revista de Investigación en Educación, n. 13(1), pp. 105-120.

Aparicio, Pablo Christian (2014). La escuela, los jóvenes y la construcción de espacios sociales incluyentes en Argentina. Restricciones y desafios. Revista del CISEN Tramas/Maepova, n. 2, pp. 1-17.

Corbí Gran, Beatriz y Pérez Nieto, Miguel Ángel (2013). The effect of absenteeism and school failure in the consumption of snuff in a simple of students in 3rd and 4th of secundary education. Health and addictions, n. 13(1), pp. 53-58.

Cutanda López, María Trinidad (2014). De los Programas de Cualificación Profesional Inicial (PCPI) a la Formación Profesional Básica: algunas consideraciones. Revista Educativa Hekademos, n. 16, pp. 69-79.

Fernández-García, Ana, Poza-Vilches, María Fátima y Fiorucci, Maximiliano (2015). Análisis Metateórico sobre el Ocio de la juventud con problemas sociales. Pedagogía Social. Revista Interuniversitaria, n. 25, pp. 119-141.

García Gómez, Soledad (2014). La investigación sobre el alumnado de los Programas de Cualificación Profesional Inicial ¿Punto de partida de la Formación Profesional Básica?. Revista de Investigación en Educación, n. 12(2), pp. 176-190.

García-Arjona, Noemí (2014). Integración social y deporte de proximidad en Francia. Un ejemplo de intervención pública en París. Gazeta de Antropología, n. 30(2), pp. $1-19$.

González González, María Teresa y Porto Currás, Mónica (2013). Programas de Cualificación Profesional Inicial: valoraciones e implicación de los alumnos de la Comunidad Autónoma de Murcia. Revista de Educación, n. Extraordinario, pp. 210-235.

Jurado de los Santos, Pedro, Olmos Rueda Patricia y Pérez Romero, Antonio (2015). Los jóvenes en situación de vulnerabilidad y los programas formativos de transición al mundo del trabajo. Educar, n. 51(5), pp. 211-224.

Márquez Vázquez, Carmen y Gualda Caballero, Estrella (2014). Absentismo escolar en Secundaria: diferencias entre nacionales e inmigrantes en la provincia de Huelva. Clave Pedagógica, n. 13, pp. 55-66.

Melendro, Miguel (2014). Transitar a la vida adulta cuando se es joven y vulnerable: estrategias de actuación en una sociedad en crisis. Metamorfosis. Revista del Centro Reina Sofía sobre Adolescencia y Juventud, n. 1, pp. 37-54.

Montserrat, Carmen y Melendro, Miguel (2017). ¿Qué habilidades y competencias se valoran de los profesionales que trabajan con adolescencia en riesgo de exclusión social? Análisis desde la acción socioeducativa. Revista Educación XX1, n. 20(2), 113-135.

Navarro-Pérez, José-Javier, Pérez-Cosin, José-Vicente y Perpiñán, Silvia (2015). El proceso de socialización de los adolescentes: entre la inclusión y el riesgo. Recomendaciones para una ciudadanía sostenible. Pedagogía Social. Revista Interuniversitaria, n. 25, pp. 143-170. 
Olmos Rueda, Patricia y Mas Torelló, Oscar (2013). Youth, Academic Failure and second chance training programmes. REOP Revista Española de Orientación y Psixopedagogía, n. 24(1), pp. 78-93.

Olmos Rueda, Patricia y Mas Torelló, Oscar (2017). Perspectiva de tutores y de empresas sobre el desarrollo de las competencias básicas de empleabilidad en el marco de los programas de formación profesional básica. Revista Educar, n. 53(2), pp. 261-284.

Orden 1409/2015, de 18 de mayo, de la Consejería de Educación, Juventud y Deporte, por la que se regulan aspectos específicos de la Formación Profesional Básica en la Comunidad de Madrid.

Organización Internacional del Trabajo (OIT). (2016). Perspectivas sociales y de empleo en el mundo 2016: tendencias del empleo juvenil.

Palomares Ruiz, Ascensión y López Sánchez, Sagrario (2013). Los Programas de Cualificación profesional inicial y la atención a la diversidad en Castilla-La Mancha. Enseñanza \& Teaching, n. 31(2), pp. 23-44.

Pérez Islas, Verónica, Velázquez Altamirano, Marina, Villanueva Solano, Anabel, Sánchez García, Lidia María y Fernández Cáceres, María del Carmen (2016). Percepción y significación de jóvenes excluidos del contexto escolar o laboral y consumo de sustancias psicoactivas. Health and Addictions, n. 16(1), pp. 19-32.

Pérez Serrano, Gloria, Poza-Vilches, María Fátima y Fernández-García, Ana (2016). Criterios para una intervención de calidad con jóvenes en dificultad social. Revista Española de Pedagogía, n. 74(263), pp. 51-69.

Real Decreto 127/2014, de 28 de febrero, por el que se regulan aspectos específicos de la Formación Profesional Básica de las enseñanzas de formación profesional del sistema educativo, se aprueban catorce títulos profesionales básicos.

Román, Marcela. (2013). Factores asociados al abandono y la deserción escolar en América Latina: Una mirada en conjunto. Revista Iberoamericana sobre Calidad, Eficacia y Cambio en Educación, n. 11(2), pp. 34-68.

Sánchez-Castañeda, Alfredo (2014). Los jóvenes frente al empleo y el desempleo: la necesaria construcción de soluciones multidimensionales y multifactoriales. Revista Latinoamericana de Derecho Social, n. 19, pp. 133-162.

Sarceda-Gorgoso, María-Carmen, Santos, María Carmen y Sanjuán Roca, María del Mar (2017). La Formación Profesional Básica: ¿alternativa al fracaso escolar?. Revista de Educación, n. 378, pp. 78-112.

Tarabini, Aina y Montes, Alejandro (2015). La agenda política contra el abandono escolar prematuro en España: la LOMCE contra las evidencias internacionales. Revista de la Asociación de Inspectores de Educación de España, n. 23, pp. 1-20.

Vallejo Peña, Alberto (2017). La generación Y ante el desafío de su inserción laboral: realidades frente a estereotipos. ARBOR Ciencia, Pensamiento y Cultura, n.193(786), pp. 1-14. 
